\title{
Black sugarcane decoction reduces rat brain ischemia
}

\author{
Ety S. Handayani*, Zainuri S. Nugraha*, Titis Nurmasitoh**, Kuswati*, \\ Dwi N. Ahsani***, and Ajeng G. Nanda****
}

-Anatomy Department of Medical
Faculty,
Islamic University of Indonesia
**Physiology Department of Medical
Faculty,
Islamic University of Indonesia
***Histology Department of Medical
Faculty,
Islamic University of Indonesia
****Medical Profession Program,
Medical Faculty,
Islamic University of Indonesia
Correspondence :
dr. Ety Sari Handayani, M.Kes
Department of Anatomy,
Faculty of Medicine,
Islamic University of Indonesia
Jalan Kaliurang Km 14,5 Yogyakarta
Indonesia
Phone: +62858 7819 2619
Email: eshyasser@ yahoo.co.id

Univ Med 2016:35:40-5

DOI: 10.18051/UnivMed.2016.v35.40-45 pISSN: 1907-3062 / eISSN: 2407-2230

This open access article is distributed under a Creative Commons Attribution-Non Commercial-Share Alike 4.0 International License

\section{BACKGROUND}

There are people in Yogyakarta, who use black sugarcane decoction (BSD) to prevent stroke. BSD contains policosanol and antioxidants. It has been proven that policosanol can reduce global ischemia in Mongolian gerbils. This study aims to evaluate the effect of BSD on brain ischemia in a rat stroke model.

\section{METHODS}

A laboratory experiment using eighteen 3-month old male Wistar rats without any defects, of 175-250 g body weight. Brain ischemia was produced by a 20 -minute bilateral carotid communis artery oclusion (BCCAO). Using a rat stroke model, brain ischemia was produced by a 20-minute BCCAO. The rats were randomized into three groups: BSD treated stroke model rats (group 1), non treated stroke model rats (group 2 ), and sham operated rats (group 3). BSD was administered by gavage for 1 week before BCCAO. Decapitation of rats was performed two hours post BCCAO. Brain tissues were stained with 2,3,5-triphenyltetrazolium chloride (TTC). Ischemic areas were analyzed using Image J softwere. Statistical analysis was conducted by one way ANOVA test.

\section{RESULTS}

The mean percentages of rat brain ischemic area differed between group $3(0.0 \pm 0.0 \%)$, group $2(3.13 \pm 0.59 \%)$ and group $1(1.15 \pm 0.47 \%) \mathrm{p}$ $=0.001)$. Post hoc test showed that there was no difference between group 3 with group 1. Instead, there was a significant difference between group 2 and the other groups.

\section{CONCLUSION}

The administration of BSD reduced rat brain ischemia after bilateral carotid artery ligation.

Keywords: Sugarcane, brain ischemia, bilateral carotid communis artery oclusion, rats 


\section{INTRODUCTION}

Scientific research shows that sugarcane contains policosanol, a mixture of long-chain alcohols, including mainly octacosanol. ${ }^{(1)}$ Preclinical trials on policosanol showed that the compound had the ability to reduce the incidence of stroke through the mechanism of inhibition of platelet aggregation and a decrease in blood cholesterol levels. ${ }^{(1,2)}$ It was the question of the benefits of policosanol as a stroke therapy that was still debatable. A clinical study showed that administration of policosanol immediately after the incidence of ischemic stroke would reduce subsequent ischemic strokes. ${ }^{(3)}$ An experimental study showed that oral policosanol pre-treatment $(50-400 \mathrm{mg} / \mathrm{kg}$ ) protected against blood-brain barrier damage induced by cerebral ischemia/ reperfusion in rats. ${ }^{(4)}$

In addition to policosanol, sugarcane also contains antioxidants, especially quercetin. In vivo, the ability of quercetin in reducing cerebral ischemia is still debated. There are controversies about the ability of quercetin to pass through the blood brain barrier. ${ }^{(5)}$ In contrast, antioxidant compounds in sugarcane could reduce oxidative stress in human HepG2 cells in vitro. ${ }^{(6,7)}$

Based on the fact that sugarcane contains policosanol and quercetin, the usage of black sugar cane decoction (BSD) as a stroke therapy in the community, and the development of a rat stroke model, in which brain ischemia was produced by a 20 -minute bilateral carotid communis artery oclusion (BCCAO). The objective of this study was evaluate the effect of $\mathrm{BSD}$ in decreasing BCCAO-induced rat brain ischemia.

\section{METHODS}

\section{Research design}

This research applied a quasi-experimental design, using a post-test for the control group. The study was conducted from September until November 2015 at the Pharmacy Laboratory, Islamic University of Indonesia.

\section{Animals and experimental procedure}

The animals used in this study were 18 male rats (Rattus norvegicus of the Wistar strain) that had met the inclusion and exclusion criteria. The rats were reared in the Pharmacy Laboratory, Inslamic University of Indonesia. Inclusion criteria for this study were healthy 3-month old male rats without any defects, of 175-250 g body weight. Determination of healthy rats was based on the physical state of the rats, i.e. those with clean, not wet or sticky bristles, active movements, and appropriate cycle of eating, drinking and sleeping. Exclusion criteria of this research were sick and dying rats during the study.

From day 1 to 7, the experimental animals were kept in cages of $40 \times 20 \times 20 \mathrm{~cm}$ for adaptation, at a density of one rat per cage. The inside temperature of the cage was set at room temperature. Lighting was arranged with a 12hour light-dark cycle. The light cycle began at $06.00 \mathrm{am}$ and dark cycle at 06:00 pm. Pellets were given every day in the morning at $06.00 \mathrm{am}$. Drinking water was provided ad libitum.

Subjects were randomized into three groups, of which each consisted of 6 rats. The groups were as follows: group 1 consisted of BSD treated stroke model rats (rats with the stroke model receiving $\mathrm{BSD}$ by gavage for 1 week before BCCAO), group 2 contained non-treated stroke model rats, and group 3 comprised sham operated rats (rats undergoing the same operation without $\mathrm{BCCAO}$ ). Brain ischemia was produced by a 20 minute BCCAO.

\section{BCCAO procedure}

BCCAO was performed on the 15th day. Stages of the ligation are as follows: a). Anesthesia. During surgery, the rats were anesthetized using 80-100 mg/kg i.m. ketamine. The rats were placed on a sterile platform and the rat rectal temperature was kept at $37 \pm 1^{\circ} \mathrm{C}$. b). Disinfection stage. This stage aims to prevent infection. Surgical swipes are done with betadine from the center of surgical site outwards (anterior surface of the rat neck). c). Incision stage. The anterior neck was opened with a midline vertical 
incision. The underlying submandibular gland was then dissected, as was also the medial side of the right sternocleidomastoid muscle to expose the common carotid artery (CCA). The arteries were then carefully separated from the vagus nerve and connective tissue. d. Ligation stage. Using a sterile nylon suture, both carotid arteries were ligated for 20 minutes. e. Subsequently analgesic therapy was administered as $0.1 \mathrm{~mL}$ $0.25 \%$ bupivacaine, at the frequency of one time/ day (bupivacaine is the analgesic recommended for the rat stroke model).

\section{Preparation of BSD}

The BSD was made based according to a modification of Asikin's protocol. (7) The policosanol dose was $200 \mathrm{mg} / \mathrm{kg} /$ day. ${ }^{(4)}$ Since $100 \mathrm{~g}$ fresh sugarcane peel contains $500 \mathrm{mg}$ policosanol, the sugarcane peel dose was $40 \mathrm{~g} /$ $\mathrm{kg} /$ day. The number of boiled sugarcane stalks were determined by the daily volume of the solution that was required. Before boiling, the black sugarcane should be stored at a temperature of $10^{\circ} \mathrm{C} .{ }^{(8)}$

\section{Preparation of the brain}

The rat brain tissues were taken at day 15 , two hours post BCCAO. Decapitation of rats was performed with a transcardial perfusion technique. Brain tissues were stained with 2,3,5triphenyltetrazolium chloride (TTC) as follows:
A 2\% TTC solution in 1x phosphate-buffered solution (PBS) was prepared (i.e. $2 \mathrm{~g}$ TTC in $100 \mathrm{ml} 1 \mathrm{x}$ PBS). The brain was cut in the coronal plane at $2 \mathrm{~mm}$ thickness, and the sliced brain was incubated in the $2 \%$ TTC solution in black boxes for 15-20 min. The TTC solution was carefully aspirated and fresh $10 \%$ PFA solution was added. The TTC solution should be protected from light and kept at room temperature. The parts of the rat brains that were studied comprised the cerebral cortex and striatum at the first slice of brain.

\section{Statistical analysis}

The relative sizes of the ischemic areas were analyzed using Image J. Statistical analysis was performed by using one-way ANOVA test .

\section{Ethical clearance}

This study was reviewed by the Ethical Clearance Committee For Preclinical Research, Faculty of Medicine, Islamic University of Indonesia.

\section{RESULTS}

The ischemic brain area appeared white in each group using image $\mathbf{J}$ software (Figure 1). The size of the ischemic area was expressed as a percentage, i.e. the ratio of the ischemic area to the total area of the brain.

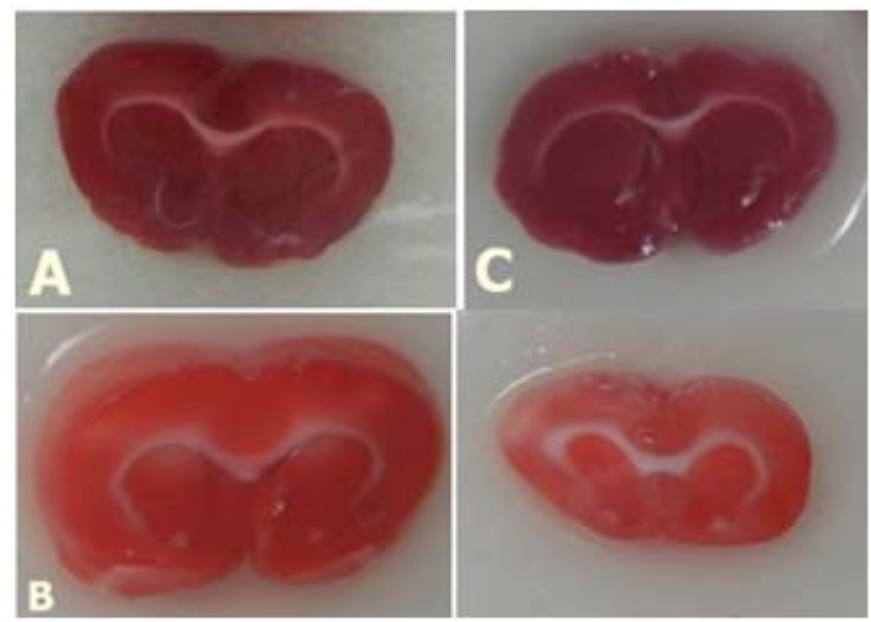

Figure 1. TTC staining results. A. Sham-operated rats, B. Non-treated stroke model rats, C. BSD-treated stroke model rats 
Table 1. Mean percentages of rat brain ischemic areas by treatment groups

\begin{tabular}{lcccc}
\hline & \multicolumn{3}{c}{ Treatment grouss } & \multirow{2}{*}{ p } \\
\cline { 2 - 5 } & $\begin{array}{c}\text { Sham-operated } \\
\text { rats }\end{array}$ & $\begin{array}{c}\text { Non-treated stroke } \\
\text { model rats }\end{array}$ & $\begin{array}{c}\text { BSD-trea ed stroke } \\
\text { model rats }\end{array}$ & p \\
\hline Rat brain ischemia(\%) & $0.00 \pm 0.00$ & $3.13 \pm 0.59$ & $1.15 \pm 0.47$ & $0.001^{*}$ \\
\hline
\end{tabular}

$\mathrm{BSD}=$ black sugarcane decoction; *statistically significant

These results indicated that there were differences in the mean percentage of rat brain ischemic area between sham-operated rats $(0.00$ $\pm 0.00 \%)$, non-treated stroke model rats $(3.13 \pm$ $0.59 \%)$ and BSD-treated stroke model rats $(1.15$ $\pm 0.47 \%)(\mathrm{p}=0.001)($ Table 1$)$. Post hoc test showed that there was no difference between the percentage of ischemic brain area in shamoperated rats and in the BSD-treated stroke model rats. Meanwhile, there was a significant difference between the group of non-treated stroke model rats and the other groups (Table 2).

\section{DISCUSSION}

Our results show that a 20-minute BCCAO can induce the occurrence of ischemic areas of the cortex and striatum. TTC staining shows ischemic areas of the brains of non-treated stroke model rats, occurring 2 hours post BCCAO. The results support the other research studies, stating that bilateral ligation of the carotid arteries for 30 minutes and reperfusion for 1 hour caused global ischemia in Sprague-Dawley rat brains and infarcted areas on TTC staining. ${ }^{(9)}$

2,3,5-Triphenyltetrazolium chloride is a water-soluble dye that binds to dehydrogenases and nicotinamide cofactors (NAD) in the mitochondria. A healthy tissue is dark red whereas damaged mitochondria in ischemic areas of the brain are white because they will not be stained by TTC..$^{(9)}$ Bilateral carotid artery ligation causes brain ischemia, resulting in necrosis and swelling of the cells, breakdown of the intracellular organelles and plasma membrane, releasing enzymes such as lactate dehydrogenase $(\mathrm{LDH})$ into the plasma ${ }^{(10)}$ where its concentration can be measured. Compared to other enzymes, lactate dehydrogenase is a more sensitive marker of brain ischemia and can be used to assess the incidence of stroke. $^{(11)}$

The period in which the enzyme levels increase in the blood varies from 8 hours to several days after the onset of stroke. Lactate dehydrogenase levels in brain tissue may reach a peak at 48-120 hours post stroke. In the first hours of stroke there are differences in lactate dehydrogenase levels between cerebral cortical and subcortical ischemic lesions. ${ }^{(11)}$

In one study, an increase was found in rat brain LDH activity under occlusion of the carotid artery for 1 hour. ${ }^{(12)}$ Similarly, in another study, there was an increase in the LDH activity in pyramidal neurons of the hippocampus and layers II, III and V of the cerebral cortex of the Mongolian gerbils after 7 minutes of ischemia. The LDH activity was variable, LDH levels beginning to appear at the $7^{\text {th }}$ minute of ligation, declining in the first 2 hours post-ligation, and returning to normal 7 days after the onset of ischemia (reperfusion period). ${ }^{(11)}$

Table 2. Post Hoc test

\begin{tabular}{ccl}
\hline & Treatment gro us & p \\
\hline Sham-operatedrats & Non-treated stroke model rats & $0.015^{*}$ \\
& BSD -treated stroke model rats & 0.184 \\
Non-treated stroke model rats & BSD-treated stroke model rats & $0.000^{*}$ \\
\hline
\end{tabular}

BSD=black sugarcane decoction; *statistically significant 
The ischemic areas of the BSD-treated stroke model rats are smaller than those of the non-treated stroke model rats. These results show that black sugarcane can be used as a neuroprotectant, since sugarcane is a source of antioxidants. ${ }^{(13)}$ The phenolic concentration in molasses is $381 \mu \mathrm{g} / \mathrm{g}$ molasses. The phenolic compounds contain cathechin (16.42 mg/g molasses) and quercetin 3-O-glucosyl-xyloxide (25.27 mg/g molasses). Catechin functions as an antioxidant, ${ }^{(14)}$ while it is known that quercetin has anti-tumoral, antithrombotic, anti-inflammatory and anti-apoptotic effects. Quercetin plays an active role in the cellular mechanisms, by acting as an inhibitor of phosphatidylinositol-3 kinase, protein kinase $\mathrm{C}$, xanthine oxidase and NADPH diaphorase, causing quercetin to have a neuroprotective effect. $^{(5)}$ The high levels of these phenolics in molasses allow the use of these compounds in the prevention and treatment of diseases caused by oxidative stress, because molasses is able to protect the cells, being even more protective than á-tocopherol. ${ }^{(6,7)}$ Phenolics in black sugarcane can inhibit lipid peroxidation in the brain so that black sugarcane can be used as a neuroprotectant. ${ }^{(15)}$

In addition, there are higher primary aliphatic acids derived from sugarcane wax that are called D-003. These compounds have antioxidant properties and mainly contain octacosanoic acid (C28). D-003 is able to inhibit plasma lipid peroxidase. ${ }^{(16,17)}$ Toxicity tests of sugarcane molasses have proven that $\mathrm{D}-003$ is safe since it does not increase LDH levels. ${ }^{(18)}$

One study found that $50-400 \mathrm{mg} / \mathrm{kg}$ policosanol protects effectively against cerebral ischemia in gerbils. ${ }^{(19)}$ The administration of 200 $\mathrm{mg} / \mathrm{kg}$ D-003 reduces cerebral edema and clinical signs in Mongolian gerbils with bilateral carotid artery ligation. ${ }^{(17)}$ Policosanol, a mixture of high molecular weight alcohols purified from sugarcane wax, has been shown to produce antiplatelet and antioxidant effects. ${ }^{(20)}$

One limitation of this study is the use of catgut threads to tie the carotid arteries. It is necessary to ensure that the ligatures on both sides are of equal strength. The results of this study can be used as a scientific basis for the community that the black sugarcane can be used as a neuroprotectant to prevent stroke. Further research is necessary to determine the therapeutic dose, toxicity of black sugarcane decoction, and development of the ligation technique using artery clamps.

\section{CONCLUSION}

This study demonstrated the neuroprotective effects of BSD in experimental brain ischaemia rats.

\section{CONFLICT OF INTEREST}

Competing interests: no relevant disclosures.

\section{ACKNOWLEDGEMENT}

This study used a grant from UPPM, Faculty medicine, Islamic University of Indonesia.

\section{REFERENCES}

1. Gnanaraj RA. Applications of sugarcane wax and it's products: a review. Int J Chemtech Res 2012;4:705-12

2. Taylor J, Rapport L, Lockwood G.B. Octacosanol in human health. Nutrition 2003;19:192-5.

3. Sánchez J, Illnait J, Mas R, et al. Effects of policosanol plus aspirin therapy on the neurological recovery and plasma oxidative markers of patients with ischemic stroke. J Pharm 2013;3:31-40.

4. Guerra YP, Cuevas VM, Ferreiro RM, et al. Effects of policosanol pre-treatment on bloodbrain barrier damage induced by ischemiareperfusion in rats. Int $\mathbf{J}$ Pharm Sci Rev Res 2015;32:1-6.

5. Dajas F, Rivera-Megret F, Blasina F, et al. Neuroprotection by flavonoids. Braz J Med Biol Res 2003;36:1613-20.

6. Valli V, Caravaca A, Nunzio M, et al. Sugar cane and sugar beet molasses, antioxidant-rich alternatives to refined sugar. J Agric Food Chem 2012;60:12508-15.

7. Asikin Y. Flavor characteristics and biological functions of Okinawan sugary and citrus materials (thesis). United Graduate School of 
Agricultural Sciences: Kagoshima University, Japan;2014.

8. Krishnakumar T, Thamilselvi C, Devadas CT. Effect of delayed extraction and storage on quality of sugarcane juice. Afr J Agric Res 2013; 8:930-5.

9. Rekabi MD, Hussein FH, Mosawi A, et al. Histopathological effects of im methionine in rat cerebral ischemia reperfusion I/ $\mathrm{R}$ injury. $\mathrm{Br} \mathrm{J}$ Med Health Res 2015;2:2394-67.

10. Chan F, Moriwaki K, Rosa MJ. Detection of necrosis by release of lactate dehydrogenase (LDH) activity. Methods Mol Biol 2013;979:6570. doi:10.1007/978-1-62703-290-2_7.

11. Shcherbak NS, Galagudza MM, Ovchinnikov DA, et al. Lactate dehydrogenase activity in the cerebral cortex and hippocampus of Mongolian gerbils in ischemic and reperfusion injuries. Neurosci Behav Physiol 2013;43:941-5.

12. Khalilov RA, Dzhafarova AM, Dzhabrailova $\mathrm{RN}$, et al. Analysis of the kinetic characteristics of lactate dehydrogenase from the rat brain during ischemia and reperfusion.Neurochem $\mathbf{J}$ 2014;8:265-70.

13. Khan SK, Tahir M, Lone KP, et al. Protective effect of Saccharum officinarum L. (sugar cane) juice on isoniazid induced hepatotoxicity in male albino mice. J Ayub Med Coll Abbottabad 2015; 27:2.
14. Chacko SM, Thambi PT, Kuttan R, et al. Beneficial effects of green tea: a literature review. Chinese Med 2010;5:13.

15. Almeida JM, Novoa AV, Linares AF, et al. Antioxidant activity of phenolics compounds from sugar cane (Saccharum officinarum L.) juice. Plant Foods Hum Nutr 2006;61:187-92.

16. Perez Y, Molina V, Mas R, et al. Comparison of in vivo effects of D-003, a mixture of high molecular weight sugarcane wax acids and grape seed extract on lipid peroxidation markers in rats. Lat Am J Pharm 2008;27:498-504.

17. Molina V, Noa M, Arruzazabala L, et al. Effect of D-003, a mixture of very-long-chain aliphatic acids purified from sugarcane wax on cerebral ischemia in Mongolian gerbils. J Med Food 2005;8:482-7. doi:10.1089/jmf.2005.8.482.

18. Rahiman F, Pool EJ. Preliminary study on the effect of sugar cane (Saccharum officinarum) molasses on steroidogenesis in testicular cell cultures. African J Food Sci 2010;4:37-40.

19. Molina V, Ravelo Y, Noa M, et al. Therapeutic effects of policosanol and atorvastatin against global brain ischaemia-reperfusion injury in gerbils. Indian J Pharm Sci 2013;75:635-41.

20. Molina V, Carbajal D, Arruzazabala ML, et al. Comparative study of policosanol and grape seed extract on platelet aggregation in rats. Rev Cienc Biol 2011;42:3-6. 